\title{
Antitumor Activity of (2E,5Z)-5-(2-Hydroxybenzylidene)-2-((4-phenoxy- phenyl)imino) thiazolidin-4-one, a Novel Microtubule-Depolymerizing Agent, in U87MG Human Glioblastoma Cells and Corresponding Mouse Xenograft Model
}

\author{
Qiu Zhang ${ }^{1}$, Xiaojun Liu ${ }^{1}$, Xiue Li ${ }^{1}$, Changlong $\mathrm{Li}^{2}$, Hongyu Zhou ${ }^{2}$, and Bing Yan ${ }^{1, *}$ \\ ${ }^{1}$ School of Chemistry and Chemical Engineering, Shandong University, Jinan 250100, China \\ ${ }^{2}$ Department of Chemical Biology and Therapeutics, St. Jude Children's Research Hospital, Memphis, Tennessee 38105, USA
}

Received April 1, 2013; Accepted May 26, 2013

\begin{abstract}
Glioblastoma is the most lethal brain cancer. In spite of intensive therapy, the prognosis of patients with glioblastoma is very poor. To discover novel therapeutic agents, we screened a combinatorial compound library containing 372 thiazolidinone compounds using U87MG human glioblastoma cells. (2E,5Z)-5-(2-hydroxybenzylidene)-2-((4-phenoxyphenyl)imino) thiazolidin4-one (HBPT) was identified as the most potent anti-glioblastoma compound. HBPT inhibits U87MG human glioblastoma cell proliferation with an $\mathrm{IC}_{50}$ of $20 \mu \mathrm{M}$, which is almost 5-fold more potent than temozolomide (a widely used drug for treating malignant glioma in the clinic). Mechanistic investigation demonstrated that HBPT is a novel microtubule-depolymerizing agent, which arrests cancer cells at the G2/M phase of the cell cycle and induces cell apoptosis. In the mouse U87MG xenograft model, HBPT elicits a robust tumor inhibitory effect. More importantly, no obvious toxicity was observed for HBPT therapy in animal experiments. These findings indicate that HBPT has the potential to be developed as a novel agent for the treatment of glioblastoma.
\end{abstract}

[Supplementary Tables: available only at http://dx.doi.org/10.1254/jphs.13064FP]

Keywords: thiazolidinone, glioblastoma, microtubule, microtubule-depolymerizing agent, U87MG human glioblastoma cell

\section{Introduction}

Gliomas are the most common types of primary brain tumor at all ages (1). Nearly two thirds of gliomas are highly malignant, with glioblastoma being the most prevalent and deadliest form in adults $(2,3)$. Patients with glioblastoma have poor prognosis. Their median survival time is approximately 16 months (2). Death from glioblastoma accounts for approximately $4 \%$ of all cancer deaths each year (1).

The standard therapy for patients with glioblastoma includes surgery with concomitant radiation therapy and chemotherapy. Temozolomide (TMZ), an alkylating

*Corresponding author. drbingyan@yahoo.com Published online in J-STAGE

doi: 10.1254/jphs.13064FP agent, is widely used as a standard first-line chemotherapy of glioblastoma (4). In U87MG human glioblastoma cells, TMZ has an $\mathrm{IC}_{50}$ value of $100 \mu \mathrm{M}$ (5). The introduction of TMZ has improved survival in patients with malignant gliomas. However, a considerable number of glioblastoma cases are refractory to TMZ $(6,7)$, and the prognosis of patients with glioblastoma is still poor. Therefore, effective treatment strategies for glioblastoma remain a challenge, and the development of novel chemotherapeutic agents is urgently needed.

Thiazolidinone derivatives have been investigated for a range of pharmacologic indications, such as antiinflammatory (8) and antimicrobial effects (9), but their anticancer effects have been less widely documented. To identify effective chemotherapeutic agents, we designed and synthesized a thiazolidinone library containing 372 compounds $(10,11)$. In a previous study, we identified 
several hit compounds that had potent cytotoxicity in non-small cell lung cancer $(10,11)$. In this study, the anti-glioblastoma activity of this compound library against U87MG human glioblastoma cells was evaluated by high-throughput cytotoxicity screening. Through highthroughput cytotoxicity screening of this compound library, (2E,5Z)-5-(2-hydroxybenzylidene)-2-((4phenoxyphenyl)imino) thiazolidin-4-one (HBPT) was identified as the most potent antitumor compound against U87MG human glioblastoma cells in vitro and it acts by depolymerizing microtubules. In the U87MG xenograft model, HBPT also showed robust tumor inhibitory effect with low toxicity. Our data suggested that HBPT has the potential to be further developed into an anticancer candidate agent for glioblastoma.

\section{Materials and Methods}

\section{Reagents}

The synthesis and characterization of the smallmolecule compound library containing 372 thiazolidinone derivatives were previously reported (11), and the structure and properties of the compounds are shown in Supplementary Table 1, available in the online version only. These compounds were used for lead compound screening in this study. The Annexin V-FITC Apoptosis Detection Kit was purchased from Nanjing Key Gen Biotech (Nanjing, Jiangsu, China). The fluorescencebased tubulin polymerization assay kit was purchased from Cytoskeleton (Denver, CO, USA). The Titer-Glo ${ }^{\circledR}$ Luminescent Cell Viability Assay kit and The Cell Titer $96^{\circledR}$ AQueous One Solution Cell Proliferation Assay (MTS) kit were purchased from Promega (Madison, WI, USA). Anti- $\alpha$-tubulin (11H10) was purchased from Cell Signaling Technology (Beverly, MA, USA). TMZ and other chemical reagents were obtained from SigmaAldrich (St. Louis, MO, USA).

\section{Cell culture and proliferation assay}

The U87MG human glioblastoma cell line was purchased from the American Type Culture Collection (Manassas, VA, USA) and grown in Dulbecco's modified Eagle's medium (DMEM) supplemented with 10\% heat-inactivated fetal calf serum (FCS), 100 units $/ \mathrm{ml}$ penicillin, and $100 \mu \mathrm{g} / \mathrm{ml}$ streptomycin. The medium and antibiotics were purchased from Invitrogen (Carlsbad, $\mathrm{CA}, \mathrm{USA}$ ). The cells were maintained in a $37^{\circ} \mathrm{C}$ incubator with $95 \%$ humidity and $5 \% \mathrm{CO}_{2}$. For library cytotoxicity screening, cell proliferation was measured using the Titer-Glo ${ }^{\circledR}$ Luminescent Cell Viability Assay (12) and MTS Cell Proliferation assay (13). Time-dependent cell viability was assessed using the trypan blue exclusion test (14). All of the experiments were repeated three times, and the average is shown in each figure. The $\mathrm{IC}_{50}$ value was defined as the drug concentration that inhibited $50 \%$ cell growth compared with the untreated controls and calculated by regression analysis.

\section{Time-dependent effects of HBPT on cell morphological changes}

To examine the time-dependent response to HBPT, U87MG cells $\left(4 \times 10^{3}\right.$ cells in $1000 \mu$ l of culture medium/ well) were seeded in 24-well flat-bottom plates and treated the next day with $20 \mu \mathrm{M}$ HBPT for various times. An equal volume of DMSO (0.1\%) was used as a control. The cell morphological changes were observed under an Olympus I X 71 phase-contrast microscope (Center Valley, PA, USA). Representative images were presented.

\section{Cell cycle analysis}

For cell cycle analysis, the cells were treated with HBPT $(20 \mu \mathrm{M})$ for various times. The cells were harvested, washed twice with ice-cold PBS, and fixed in $70 \%$ ethanol at $-20^{\circ} \mathrm{C}$ for $30 \mathrm{~min}$. The cells were then washed with PBS, incubated with $10 \mu \mathrm{g} / \mathrm{ml}$ RNase A at $37^{\circ} \mathrm{C}$ for $30 \mathrm{~min}$, and stained with $50 \mu \mathrm{g} / \mathrm{ml}$ propidium iodide (PI) solution. The stained cells were analyzed using a FACScan flow cytometer (BD Bioscience, Franklin Lakes, NJ, USA) and ModFit LT software.

\section{FACScan analysis by Annexin V staining}

Flow cytometry analysis was employed to detect apoptosis using an Annexin V-FITC Apoptosis Detection Kit according to the manufacturer's instruction. Briefly, cells were treated with $20 \mu \mathrm{M}$ HBPT for various times. After double staining with Annexin V-FITC and PI, apoptosis was analyzed by flow cytometry. Early apoptosis was defined as Annexin $\mathrm{V}^{+} / \mathrm{PI}^{-}$staining and late apoptosis was defined as Annexin $\mathrm{V}^{+} / \mathrm{PI}^{+}$staining, as determined by the BD FACScan flow cytometer.

\section{Tubulin polymerization assay}

Tubulin polymerization was determined with the polymerization assay kit (fluorescence based) as specified by the manufacturer. Tubulin was purified from bovine brain and was $>99 \%$ pure. Each well of the assay plate contained $5 \mu \mathrm{l}$ control buffer ( $5 \%$ DMSO in general tubulin buffer) or test compounds at the indicated concentrations, which were pre-warmed at $37^{\circ} \mathrm{C}$ for 1 min. In total, $50 \mu$ tubulin solution was rapidly pipetted into each well, and the polymerization dynamics of tubulin was monitored through measuring the change in fluorescence every $2 \mathrm{~min}$ for $42 \mathrm{~min}$ at $37^{\circ} \mathrm{C}$ with a 1420 Multi-label Counter (Perkin Elmer, Wellesley, MA, USA). 
Immunofluorescence staining for microtubule structure

Cells were plated on coverslips that were placed in 6 -well plates. After $24 \mathrm{~h}$ of incubation, DMSO, colchicine $(10 \mu \mathrm{M})$, or HBPT $(20 \mu \mathrm{M})$ was added to the wells. After $14 \mathrm{~h}$ of incubation, the cells were washed with PBS and fixed with $4 \%$ formaldehyde in PBS at room temperature for $15 \mathrm{~min}$. After being washed three times with PBS, the cells were incubated in methanol at $-20^{\circ} \mathrm{C}$ for $10 \mathrm{~min}$. The cells were washed with PBS again, incubated with blocking buffer for $60 \mathrm{~min}$, and incubated with a rabbit anti- $\alpha$-tubulin antibody overnight at $4{ }^{\circ} \mathrm{C}$. After being washed with PBS, the cells were incubated with a fluorescein isothiocyanate-conjugated fragment of goat anti-rabbit secondary antibody (ZSGB-BIO) at room temperature for $1 \mathrm{~h}$. After being washed with PBS, the cells were examined with fluorescence microscopy (Olympus Optical Co., Ltd., Tokyo).

\section{In vivo glioblastoma xenograft study}

The inhibitory effect of HBPT on human glioblastoma U87MG xenografts was examined in nude mice. All procedures involving animals and their care were approved by the Institutional Animal Care and Use Committee of the Shandong University.

Four-week-old female nude mice (BALB/c) were obtained from Vital River Research Animal Services (Beijing, China). Subcutaneous tumors were established in by inoculation of $5 \times 10^{6} \mathrm{U} 87 \mathrm{MG}$ cells into the dorsal flank of each mouse. When tumors grew to 3 to $5 \mathrm{~mm}$ in diameter, animals were randomly divided into five groups and subjected to the following treatment by i.p. injection: a) Vehicle control (5.7\% DMSO, 9.6\% Cremophor EL, and $9.6 \%$ ethanol); b) $40 \mathrm{mg} / \mathrm{kg} \mathrm{HBPT}$; c) $60 \mathrm{mg} / \mathrm{kg}$ HBPT; and d) $80 \mathrm{mg} / \mathrm{kg}$ TMZ. HBPT was administered for 15 consecutive days and TMZ was administered on day $1,2,3,4$, and 5 according to its guideline for clinical application. Tumors and body weights were measured during treatment every other day. Two perpendicular diameters of tumors were measured with a digital caliper by the same investigator. Tumor volume was calculated according to a standard formula: $\left(\right.$ width $^{2} \times$ length) $/ 2$. Tumor growth inhibitory (TGI) rate was assessed by determining \% (1-T/C), [1- (mean tumor volume of treated group on day $\mathrm{x} /$ mean tumor volume of control group on day $\mathrm{x}$ ) $] \times 100$. Animal body weight was measured as an index of toxicity. Body weight change was calculated by the following equation: (body weight at conclusion of the study) - (body weight at commencement). At the end of experiment, animals were euthanized and sacrificed.

Major organs (including heart, liver, spleen, lung, kidney, and intestine) were collected and fixed overnight in $10 \%$ formalin and then transferred to $70 \%$ ethanol.
Four- $\mu \mathrm{m}$-thick, paraffin-embedded tissue sections were stained with $H \& E$ by standard procedures. Major organs from three mice per group were examined using electron microscopy by a veterinary pathologist. Blood from each mouse was analyzed for hematopoietic indices. Representative images are shown.

\section{Statistical analysis}

The results were expressed as the means \pm standard deviation (S.D.). Student's $t$-test was used to determine the statistical significance between control and test groups. A $P$-value less than 0.05 was considered to be statistically significant.

\section{Results}

\section{Identification of the novel anti-glioblastoma compound $H B P T$}

To identify effective chemotherapeutic agents, we designed and synthesized a thiazolidinone library containing 372 compounds $(10,11)$. In this study, the anti-glioblastoma activity of this compound library against U87MG human glioblastoma cells was evaluated by high-throughput cytotoxicity screening. Screening was carried out in two stages: primary screening and dose-response determination. In the primary screening of the library, U87MG human glioblastoma cells were exposed to $5 \mu \mathrm{M}$ of the compounds for $48 \mathrm{~h}$, and cell viability was determined with the Titer-Glo ${ }^{\circledR}$ Luminescent Cell Viability Assay. Compounds that exhibited potent toxicity against cancer cells were selected for the dose-response determination. As a result, eight compounds were selected for further dose-response determinations. U87MG human glioblastoma cells were exposed to compounds at varying doses for $48 \mathrm{~h}$, followed by the MTS assay. The dose-response studies showed that all 8 compounds exhibited better potency ( $\mathrm{IC}_{50}$ values ranged from 19.6 to $74.5 \mu \mathrm{M}$ ) than TMZ ( $\mathrm{IC}_{50}$ value was approximately $100 \mu \mathrm{M}$ in U87MG cells (5)) (Fig. 1: A and B). Among them, HBPT was the most potent compound, with an $\mathrm{IC}_{50}$ value of 19.6 $\mu \mathrm{M}$. Compared with TMZ, the widely used standard first-line chemotherapy of glioblastoma, HBPT was almost 5-fold more potent. Therefore, HBPT was selected for further investigation.

To further quantify the cytotoxicity of HBPT, U87MG cells were treated with $20 \mu \mathrm{M}$ HBPT and evaluated with a trypan blue exclusion test, and cellular morphological changes were also observed. A strong increase in cell death was found $12-72 \mathrm{~h}$ after treatment with 20 $\mu \mathrm{M}$ HBPT (Fig. 2A). Time-dependent morphological features were observed in the presence of HBPT using a phase-contrast microscope (Fig. 2B). HBPT treatment 


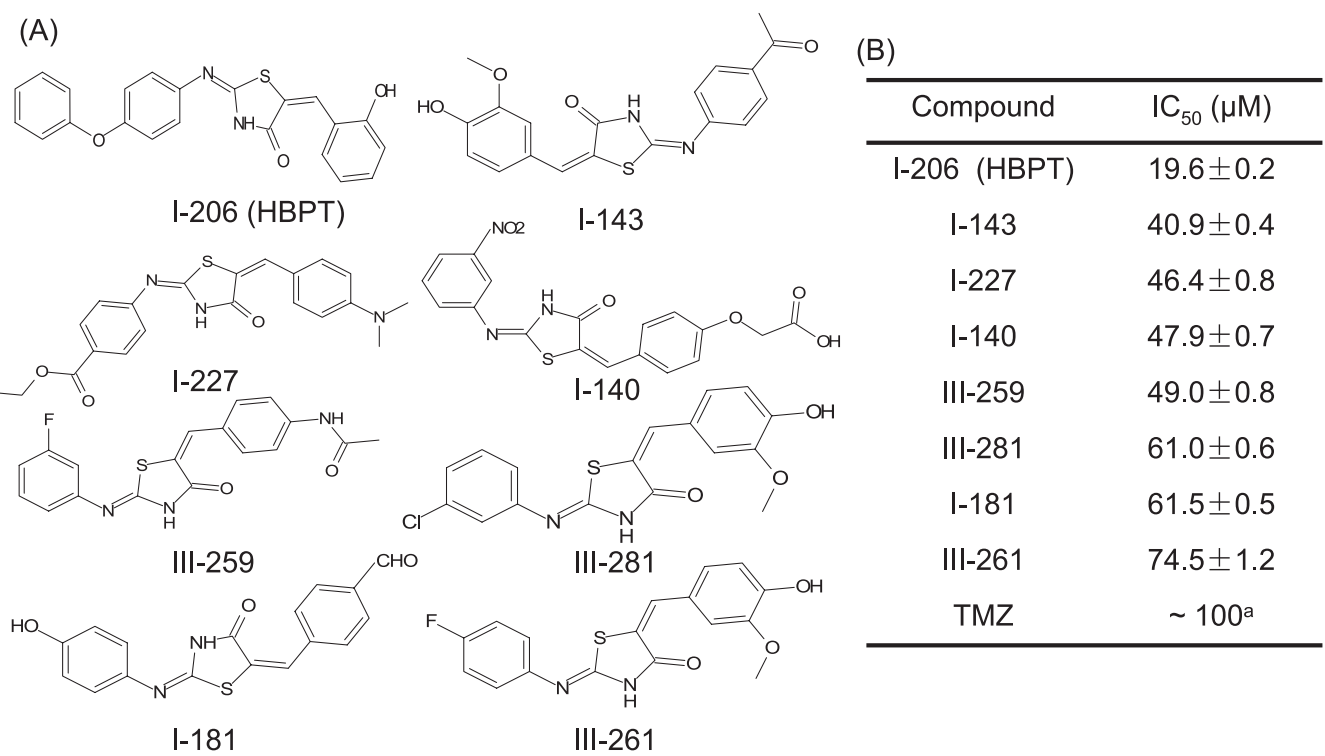

Fig. 1. Cytotoxicity of compounds in U87MG human glioblastoma cells. In panel A, chemical structures of thiazolidinone compounds were shown. In panel B, U87MG cells were cultured in the presence of compounds for $48 \mathrm{~h}$, and cell proliferation was determined by the MTS assay. $\mathrm{IC}_{50}$ was calculated. The data are expressed as the mean \pm S.D. of three independent experiments.

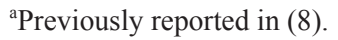

(A)

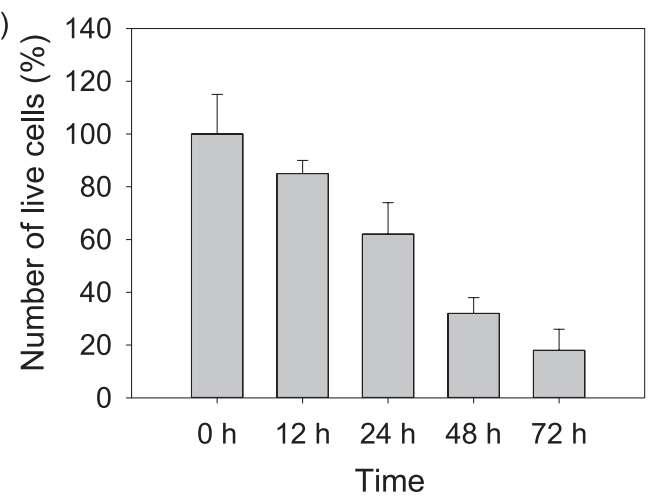

(B)
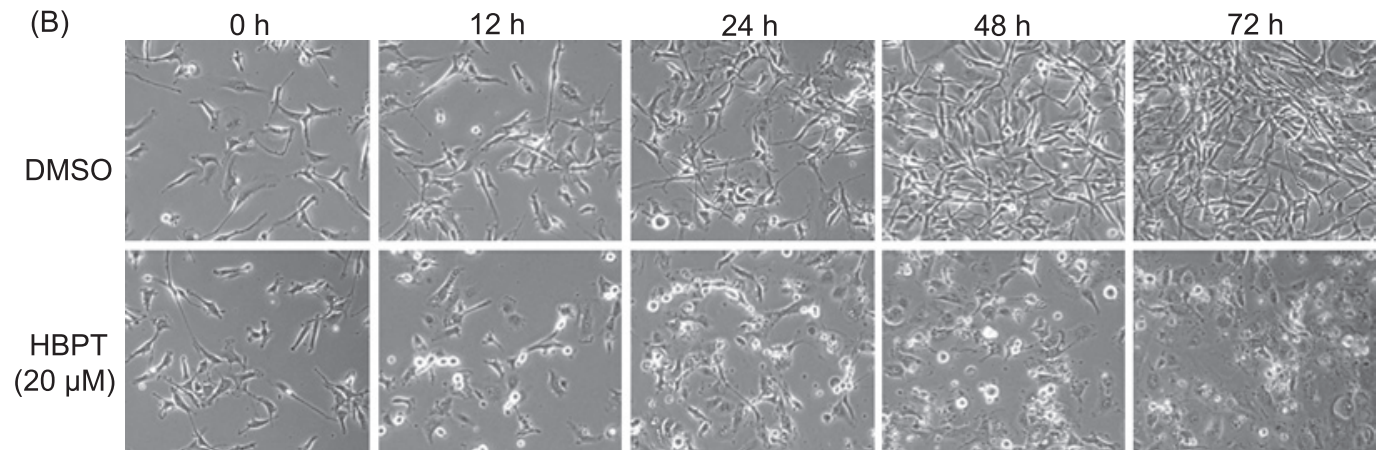

Fig. 2. Cytotoxicity of HBPT in U87MG cells. In panel A, the time-dependent effect of HBPT (20 $\mu \mathrm{M})$ on U87MG cell death was examined using the trypan blue exclusion test. In panel B, time-dependent morphological features of U87MG cells are shown in the presence of $20 \mu \mathrm{M}$ HBPT. The magnification is $200 \times$. 


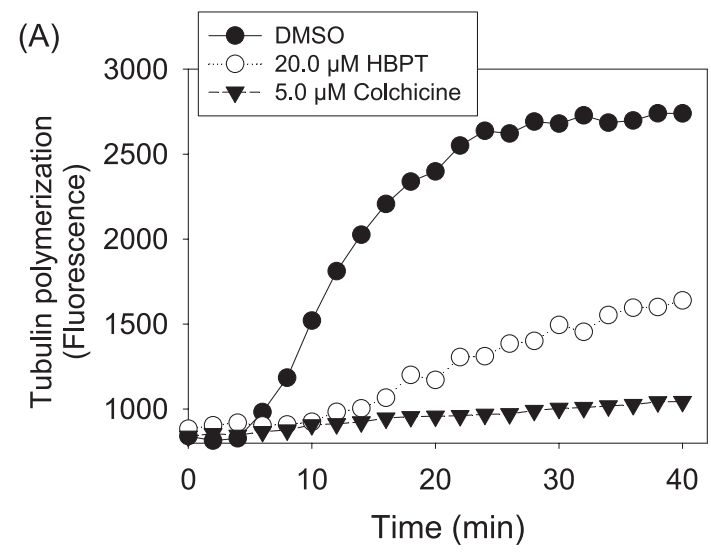

(B)

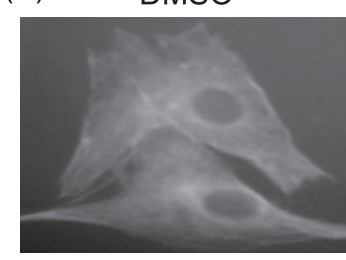

HBPT $(20 \mu \mathrm{M})$

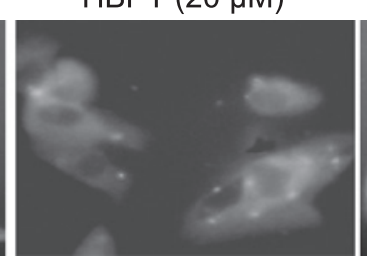

Colchicine $(5 \mu \mathrm{M})$

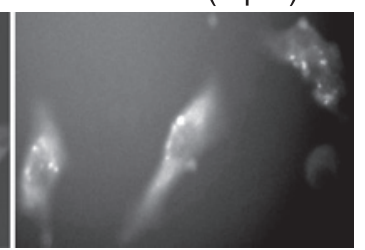

Fig. 3. Inhibitory effect of HBPT on microtubule assembly. In panel A, the inhibitory effect of HBPT on microtubule assembly was examined in a cell-free system. An in vitro polymerization assay was performed in the presence of DMSO, HBPT, or colchicine. See Materials and Methods for specifics. In panel B, cellular microtubule networks in U87MG cells after treatment with DMSO, HBPT, or colchicine for $14 \mathrm{~h}$. Cellular microtubules are detected by anti- $\alpha$-tublin antibody. Representative images are shown from three independent experiments. The magnification is $600 \times$. caused the cells to become round and become detached. Furthermore, the cellular morphological changes were correlated with the growth-inhibiting activities of HBPT, as shown in Fig. 2A. Taken together, these data suggested that HBPT had cytotoxic effects on U87MG cells in a time-dependent manner.

\section{HBPT inhibits microtubule assembly}

Microtubules are composed of $\alpha$ - and $\beta$-tubulin subunits. Their major function is to equally partition duplicate chromosomes into daughter cells through spindle formation during mitosis of the cell cycle (15). Microtubule-interfering agents perturb this dynamic process and therefore kill tumor cells. The microtubule-interfering agents can be classified into microtubule-destabilizing agents that inhibit microtubule assembly, such as colchicine and vincristine, and microtubule-stabilizing agents that disrupt microtubule disassembly, such as paclitaxel and docetaxel (15).

We previously discovered that some thiazolidinone compounds target microtubules (16); thus, we tested whether HBPT could directly affect the microtubule assembly-disassembly process. In a cell-free system, $\alpha$ - and $\beta$-tubulin subunits dimerized and self-assembled to form cylindrical microtubules in a time-dependent manner (Fig. 3A). HBPT inhibited tubulin polymerization in a similar manner as colchicine (Fig. 3A).

The HBPT inhibition of microtubule formation was further examined in live cells by immunofluorescence microscopy. U87MG cells treated with DMSO for $14 \mathrm{~h}$ displayed a dense and complex network of microtubules that were evenly distributed in the cytoplasm (Fig. 3B). In contrast, when cells were incubated with $20 \mu \mathrm{M}$ HBPT or $5 \mu \mathrm{M}$ colchicine for $14 \mathrm{~h}$, the microtubule network in cells was disrupted (Fig. 3B). These results were consistent with the data from the in vitro tubulin polymerization assay (Fig. 3A). Taken together, HBPT can inhibit microtubule assembly in a similar manner as colchicine, which is a well-known microtubuledepolymerizing agent (15).

\section{HBPT induces cell cycle arrest and apoptosis}

By suppressing microtubule dynamics, microtubuletargeting agents cause cell cycle arrest at the $\mathrm{G} 2 / \mathrm{M}$ phase and apoptosis (17). The ability of HBPT to induce cell cycle arrest in U87MG cells was examined by flow cytometry. As shown in Fig. 4A, treatment with HBPT $(20 \mu \mathrm{M})$ induced a major shift of the cells from the G0/G1 phase to the G2/M phase over time (Fig. 4A). These data suggested that HBPT treatment caused cell cycle arrest at the $\mathrm{G} 2 / \mathrm{M}$ phase in a time-dependent manner. HBPT-induced cell apoptosis was next evaluated by an Annexin V-PI staining assay. Our results showed a time-dependent increase in apoptotic U87MG cells after HBPT treatment (Fig. 4: B and C). Taken together, these data demonstrated that HBPT treatment induced G2/M arrest and apoptosis in U87MG cells, as expected from a tubulin-targeting agent $(17,18)$. These data provide further evidence that HBPT is a microtubule inhibitor that causes cell cycle G2/M arrest and apoptosis by depolymerizing microtubules. 
(A)
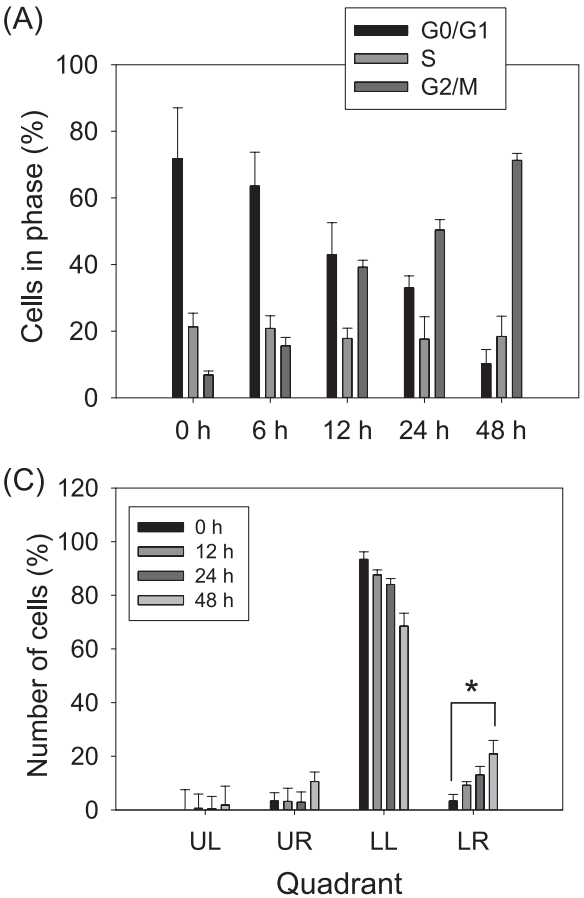

(B)

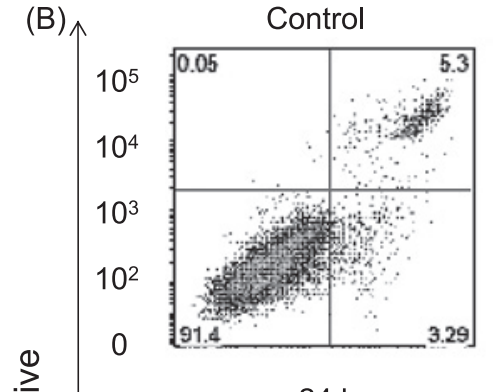

$\stackrel{0}{0}$
$\frac{1}{0}$
0
$\frac{0}{1}$
$\frac{1}{2}$

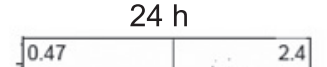

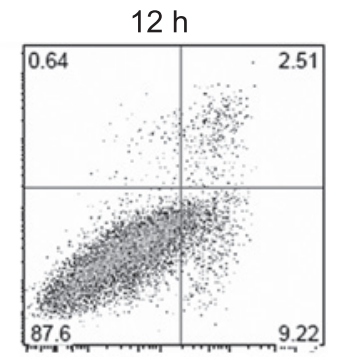
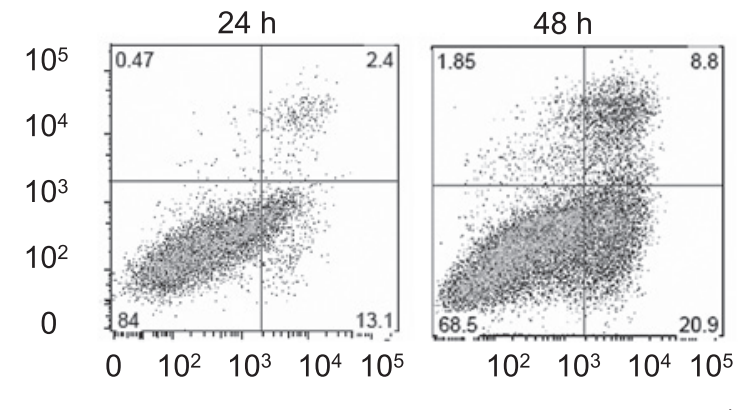

Annexin V-positive

Fig. 4. HBPT induced cell cycle arrest and apoptosis in U87MG cells. In panel A, after treatment with HBPT (20 $\mu \mathrm{M})$ for various times, cells were stained with propidium iodide and analyzed by FACScan flow cytometry. In panel B, after treatment with HBPT $(20 \mu \mathrm{M})$ for various times, cells were stained with Annexin V-FITC and propidium iodide and analyzed by FACScan flow cytometry. In panel $\mathrm{C}$, the percentages of cell numbers in each quadrant are plotted based on panel B. The results are presented as the mean \pm S.D. of three independent experiments. *Significantly different from $0 \mathrm{~h}$ at $P<0.05$. PI: propidium iodide, UL: upper left quadrant, UR: upper right quadrant, LL: lower left quadrant, LR: lower right quadrant.

\section{HBPT inhibit human U87MG xenograft growth without obvious toxicity}

HBPT's in vivo antitumor efficacy was tested on a human U87MG xenograft model in BALB/c nude mice. For HBPT, two doses of $40 \mathrm{mg} / \mathrm{kg}$ per day and $60 \mathrm{mg} / \mathrm{kg}$ per day were chosen based on our previous maximum tolerated dose (MTD) studies (data not shown). TMZ was used as a positive control. TMZ was administrated with a dose of $80 \mathrm{mg} / \mathrm{kg}$ on five consecutive days based on the literature $(19,20)$.

After 15 daily doses treatment, HBPT showed 51.4\% inhibition of tumor volume in the low-dose HBPT group (40 $\mathrm{mg} / \mathrm{kg}$ per day), and $73.2 \%$ inhibition in the high-dose HBPT group (60 mg/kg per day) (Fig. 5A). These data demonstrated that HBPT significantly inhibited tumor growth in a dose-dependent manner. Compared to the group treated with TMZ at dose of 80 $\mathrm{mg} / \mathrm{kg}$ per day (TGI is $66.6 \%$ ), high-dose HBPT showed higher tumor inhibitory effect (Fig. 5A). Consistent with in vitro data, HBPT treatment caused apoptosis of tumor cells in the U87MG xenograft mouse model as determined by TUNEL staining (data not shown). More importantly, no visible toxicity of HBPT was observed during the treatment. Several toxicity indicators includ- ing body weight during treatment, hematopoietic indices, and histopathologic examinations on heart, liver, spleen, lung, kidney, and intestine at the end point of experiment were investigated. HBPT treatment did not cause body weight loss (Fig. 5B) or abnormality in hematopoietic indices (Supplementary Table 2: available in the online version only). Furthermore, no obvious pathological changes were observed in these organs after HBPT treatment (Fig. 5C). These data suggest that HBPT at 40 or 60 $\mathrm{mg} / \mathrm{kg}$ is a well-tolerated, effective dose for BALB/c mice.

\section{Discussion}

Microtubules are an attractive target for chemotherapeutic agents, and many well-known tubulin-depolymerizing agents, such as ixabepilone and vinca alkaloids, are FDA-approved drugs for cancer therapy (19). Recently, targeting microtubules has also been recognized as a promising approach for the treatment of glioblastoma $(21,22)$. Several compounds, such as COBRA-1 (a rationally designed epoxy-THF-containing compound) (23), CP248 (a derivative of exisulind) (24), and TTI-237 (a novel chalcone derivative) (25), have 
(A)

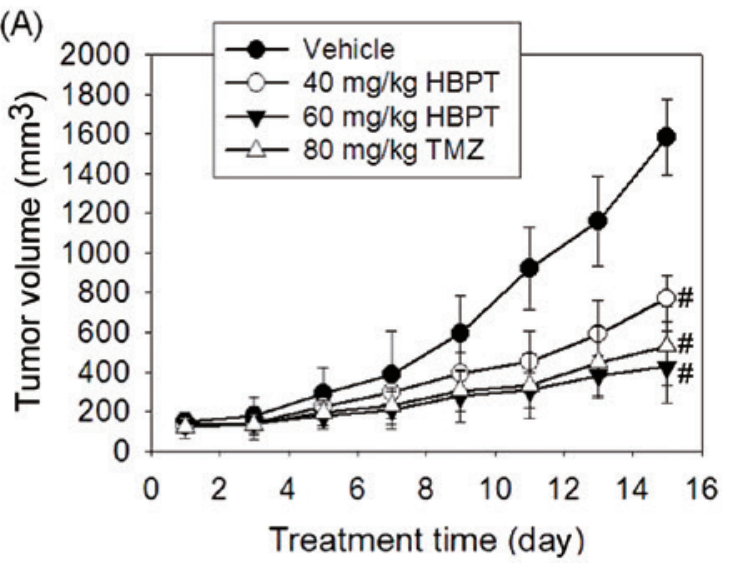

(C)

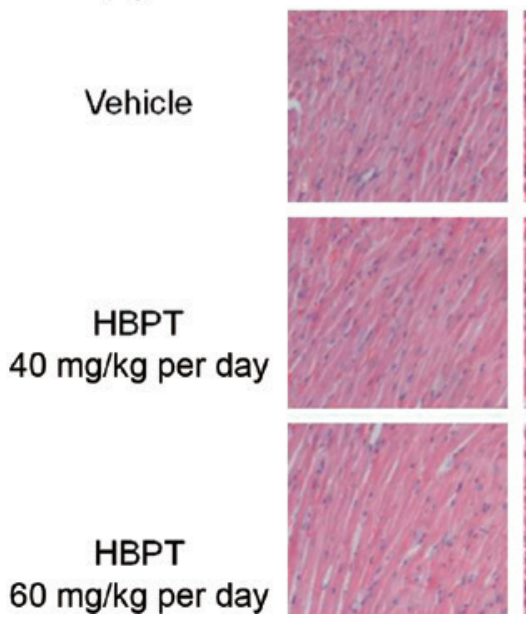

Spleen

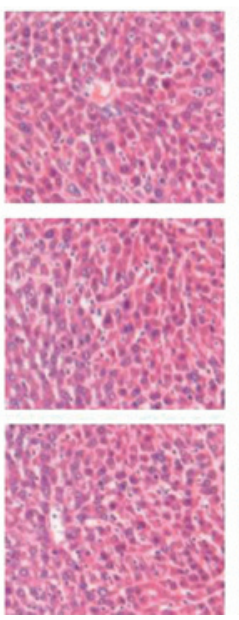

(B)

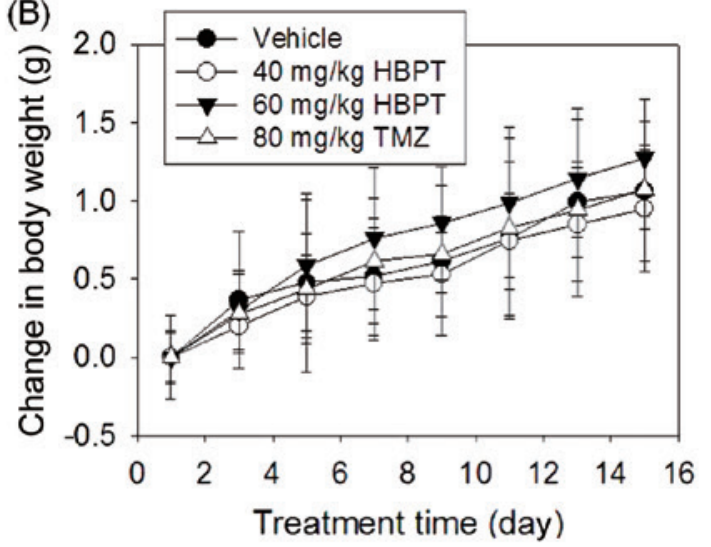

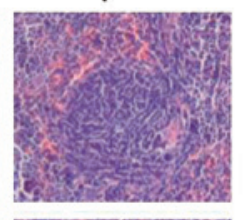
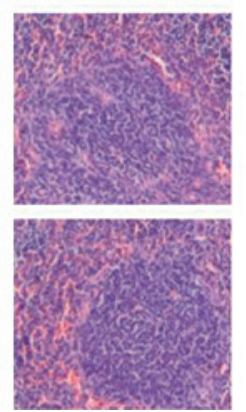

Lung
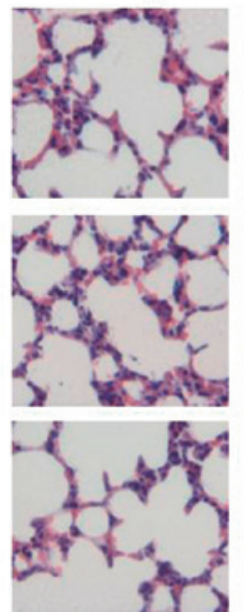

Kidney
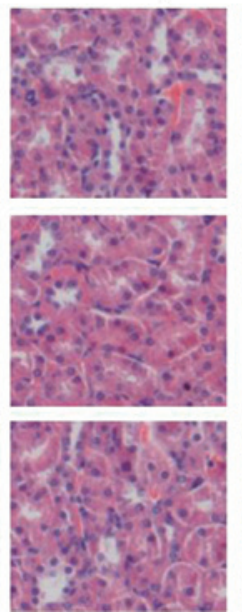

Intestine

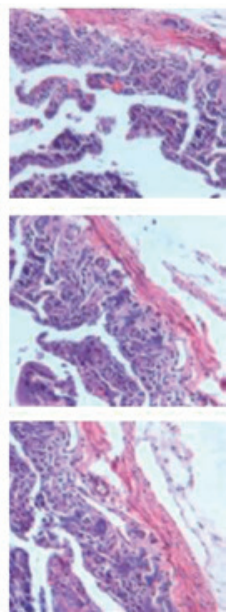

Fig. 5. Antitumor activity of HBPT in vivo. Mice with established U87MG tumor xenografts (7 mice per group) were treated with HBPT (40 mg/kg per day, $60 \mathrm{mg} / \mathrm{kg}$ per day) or solvent vehicle daily for 15 days by i.p. injection. TMZ ( $80 \mathrm{mg} / \mathrm{kg}$ per day) was dosed on day 1, 2, 3, 4, and 5 by i.p. injection. In panel A, tumor volume was monitored every two days, and was presented as the mean \pm S.D. from each group. "Significantly different from the vehicle group at $P<0.05$. In panel B, mouse body weight was monitored every two days. Body weight change was calculated by the following equation: (body weight at conclusion of the study) - (body weight at commencement). In panel C, histophathologic examination (H\&E staining) on heart, liver, spleen, lung, kidney, and intestine was done in the mice bearing U87MG tumor xenografts at the end of the experiment. Magnification is $200 \times$.

shown anti-glioblastoma activity by depolymerizing microtubules in preclinical studies. In this study, we identified the novel thiazolidinone compound HBPT as a novel microtubule-depolymerizing agent. By depolymerizing microtubules, HBPT caused the accumulation of cells at the G2/M phase and apoptosis. These results are consistent with other microtubule depolymerizing agents $(17,18)$. More importantly, HBPT has a potent cytotoxicity effect on U87 glioblastoma cells both in vitro and in vivo.

TMZ, a DNA-alkylating agent, has been widely used for treating glioblastoma. However, resistance develops quickly and with high frequency (26). In the present study, one of the most impressive effects of HBPT is that it has a more potent anti-glioblastoma effect than
TMZ both in vivo and in vitro. In U87MG cells, the $\mathrm{IC}_{50}$ value of HBPT is almost 5 -fold lower than that of TMZ. Furthermore, HBPT elicits more potent activity (TGI is $73.2 \%$ when dosed at $60 \mathrm{mg} / \mathrm{kg}$ per day) than TMZ (TGI is $66.6 \%$ when dosed at $80 \mathrm{mg} / \mathrm{kg}$ per day). As it is still pioneer experiments in animals, modification of the treatment regimen might improve the higher therapeutic efficacy.

Microtubule-targeting agents suppress microtubule dynamics, leading to disruption of the mitotic spindle in dividing cells, cell cycle arrest at the M phase, and late apoptosis. During this process, many signaling pathways are activated, such as mitotic spindle assembly checkpoint activation, Bcl-2 phosphorylation, and Jun NH2terminal kinase (JNK) activation, which ultimately lead 
to apoptosis (17). Among them, JNK activation is a hallmark event for microtubule-interfering agents such as paclitaxel, vinblastine, vincristine, docetaxel, and nocodazole (27). In our previous study, one thiazolidinone compound in this library was found to depolymerize microtubules and induce JNK activation (16). As a microtubule-depolymerizing agent, HBPT may trigger the apoptosis of cells in mitotic cell cycle arrest by inducing JNK activation.

Dose-limiting toxicity has become one big road blocker for effective cancer therapy (28). Microtubuletargeting drugs usually cause serious side effects in patients since they can block mitotic microtubule formation in cells $(29,30)$, which greatly limited their clinical application. Another important feature of HBPT is the low toxicity in animals in our experimental conditions. All of the mice treated with HBPT survived with no loss of their body weight, no abnormality of hematopoietic indices, and no damage on heart, liver, spleen, lung, kidney, and intestine during the treatment. These in vivo low toxicity profile of HBPT was consistent with the cancer-selectivity of 4-thiazolidinones compounds in vitro (11). 4-Thiazolidinones substituted in the 2-position were highly toxic to non-small cell lung cancer (NSCLC) H460 cells yet with much less toxicity to normal human fibroblast (NHFBs). As tubulin-targeting agents, the molecular mechanisms of their selective anti-tumor effects require further investigation.

In summary, these findings indicate that thiazolidinone may represent a novel type of promising microtubuledepolymerizing agent with the potential for use in glioblastoma chemotherapy.

\section{Acknowledgment}

This research was supported by the National Natural Science Foundation of China (90913006).

\section{References}

1 Desai A, Vyas T, Amiji M. Cytotoxicity and apoptosis enhancement in brain tumor cells upon coadministration of paclitaxel and ceramide in nanoemulsion formulations. J Pharm Sci. 2008;97: 2745-2756.

2 Reardon DA, Rich JN, Friedman HS, Bigner DD. Recent advances in the treatment of malignant astrocytoma. J Clin Oncol. 2006;24:1253-1265.

3 Bobola MS, Varadarajan S, Smith NW, Goff RD, Kolstoe DD, Blank A, et al. Human glioma cell sensitivity to the sequencespecific alkylating agent methyl-lexitropsin. Clin Cancer Res. 2007;13:612-620.

4 Djedid R, Kiss R, Lefranc F. Targeted therapy of glioblastomas: a 5-year view. Therapy. 2009;6:351-370.

5 Kanzawa T, Bedwell J, Kondo Y, Kondo S, Germano IM. Inhibition of DNA repair for sensitizing resistant glioma cells to temozolomide. J Neurosurg. 2003;99:1047-1052.

6 Hegi ME, Diserens AC, Gorlia T, Hamou MF, De Tribolet $\mathrm{N}$, Weller M, et al. MGMT gene silencing and benefit from temozolomide in glioblastoma. N Engl J Med. 2005;352: 997-1003.

7 Ravizza R, Cereda E, Monti E, Gariboldi MB. The piperidine nitroxide Tempol potentiates the cytotoxic effects of temozolomide in human glioblastoma cells. Int J Oncol. 2004;25: $1817-1822$.

8 Ottanà R, Maccari R, Barreca ML, Bruno G, Rotondo A, Rossi A, et al. 5-Arylidene-2-imino-4-thiazolidinones: design and synthesis of novel anti-inflammatory agents. Bioorg Med Chem. 2005; 13:4243-4252.

9 Vicini P, Geronikaki A, Anastasia K, Incerti M, Zani F. Synthesis and antimicrobial activity of novel 2-thiazolylimino-5-arylidene4-thiazolidinones. Bioorg Med Chem. 2006;14:3859-3864.

10 Teraishi F, Wu S, Zhang L, Guo W, Davis JJ, Dong F, et al. Identification of a novel synthetic thiazolidin compound capable of inducing c-Jun NH2-terminal kinase-dependent apoptosis in human colon cancer cells. Cancer Res. 2005;65:6380-6387.

11 Zhou HY, Wu SH, Zhai SM, Liu AF, Sun Y, Li RS, et al. Design, synthesis, cytoselective toxicity, structure-activity relationships, and pharmacophore of thiazolidinone derivatives targeting drug-resistant lung cancer cells. J Med Chem. 2008;51:12421251.

12 Gaither A, Porter D, Yao Y, Borawski J, Yang G, Donovan J, et al. A Smac mimetic rescue screen reveals roles for inhibitor of apoptosis proteins in tumor necrosis factor- $\alpha$ signaling. Cancer Res. 2007;67:11493.

13 Li YC, Park MJ, Ye SK, Kim CW, Kim YN. Elevated levels of cholesterol-rich lipid rafts in cancer cells are correlated with apoptosis sensitivity induced by cholesterol-depleting agents. Am J Pathol. 2006;168:1107-1118.

14 Kim KH, Park JY, Jung HJ, Kwon HJ. Identification and biological activities of a new antiangiogenic small molecule that suppresses mitochondrial reactive oxygen species. Biochem Biophys Res Commun. 2011;404:541-545.

15 Jordan MA, Wilson L. Microtubules as a target for anticancer drugs. Nat Rev Cancer. 2004;4:253-265.

16 Li LW, Zhang Q, Liu AF, Li XE, Zhou HY, Liu Y, et al. Proteome interrogation using nanoprobes to identify targets of a cancerkilling molecule. J Am Chem Soc. 2011;133:6886-6889.

17 Mollinedo F, Gajate C. Microtubules, microtubule-interfering agents and apoptosis. Apoptosis. 2003;8:413-450.

18 Perez EA. Microtubule inhibitors: Differentiating tubulininhibiting agents based on mechanisms of action, clinical activity, and resistance. Mol Cancer Ther. 2009;8:2086-2095.

19 Kato Y, Holm DA, Okollie B, Artemov D. Noninvasive detection of temozolomide in brain tumor xenografts by magnetic resonance spectroscopy. Neuro Oncol. 2010;12:71-79.

20 Newlands E, Stevens M, Wedge S, Wheelhouse R, Brock C. Temozolomide: a review of its discovery, chemical properties, pre-clinical development and clinical trials. Cancer Treat Rev. 1997;23:35-61.

21 Katsetos CD, Dráberová E, Legido A, Dumontet C, Dráber P. Tubulin targets in the pathobiology and therapy of glioblastoma multiforme. I. class III $\beta$-tubulin. J Cell Physiol. 2009;221: 505-513.

22 Katsetos CD, Dráberová E, Legido A, Dráber P. Tubulin targets in the pathobiology and therapy of glioblastoma multiforme. II. 
$\gamma$-tubulin. J Cell Physiol. 2009;221:514-520.

23 Jan ST, Mao C, Vassilev AO, Navara CS, Uckun FM. COBRA-1, a rationally-designed epoxy-THF containing compound with potent tubulin depolymerizing activity as a novel anticancer agent. Bioorg Med Chem Lett. 2000;10:1193-1197.

24 Yoon JT, Palazzo AF, Xiao D, Delohery TM, Warburton PE, Bruce JN, et al. CP248, a derivative of exisulind, causes growth inhibition, mitotic arrest, and abnormalities in microtubule polymerization in glioma cells. Mol Cancer Ther. 2002;1:393.

25 Beyer CF, Zhang N, Hernandez R, Vitale D, Lucas J, Nguyen T, et al. TTI-237: a novel microtubule-active compound with in vivo antitumor activity. Cancer Res. 2008;68:2292.

26 Shi L, Chen J, Yang J, Pan T, Zhang S, Wang Z. MiR-21 protected human glioblastoma U87MG cells from chemotherapeutic drug temozolomide induced apoptosis by decreasing Bax/Bcl-2 ratio and caspase-3 activity. Brain Res. 2010;1352:255-264.

27 Gajate C, Barasoain I, Andreu JM, Mollinedo F. Induction of apoptosis in leukemic cells by the reversible microtubuledisrupting agent 2-methoxy-5-(2', 3', 4'-trimethoxyphenyl)-2, 4, 6-cycloheptatrien-1-one: protection by $\mathrm{Bcl}-2$ and $\mathrm{Bcl}-\mathrm{XL}$ and cell cycle arrest. Cancer Res. 2000;60:2651-2659.

28 Kaelin WG. The concept of synthetic lethality in the context of anticancer therapy. Nat Rev Cancer. 2005;5:689-698.

29 Schmidt M, Bastians H. Mitotic drug targets and the development of novel anti-mitotic anticancer drugs. Drug Resist Updat. 2007;10:162-181.

30 Lee JJ, Low JA, Croarkin E, Parks R, Berman AW, Mannan N, et al. Changes in neurologic function tests may predict neurotoxicity caused by ixabepilone. J Clin Oncol. 2006;24:20842091. 